\title{
Joint Effusion, CTCAE 5.0
}

National Cancer Institute

\section{Source}

National Cancer Institute. Joint Effusion, CT CAE 5.0. NCI Thesaurus. Code C146760.

A disorder characterized by excessive fluid in a joint, usually as a result of joint inflammation. 